\title{
GOING BACK TO EINSTEIN 1907 IN THE MODERN COMPUTER AGE
}

Otto E. Rossler ${ }^{1 *}$, Yaël Kolb ${ }^{2}$, Andrei Ujica ${ }^{3}$

${ }^{*}$ Faculty of Science, University of Tübingen, Auf der Morgenstelle 8, 72076 Tübingen, Germany,

${ }^{2,3}$ University of Design (HfG), Lorenzstraße 15, 76135 Karlsruhe, Germany

*1Email: oeross00@yahoo.com

*Corresponding Author: -

E-mail ID: oeross00@yahoo.com

\begin{abstract}
: -
Einstein's equivalence principle of 1907 amounts to a mental analog computer that is ready for simulation in the modern computer age. Several new fundamental implications do already come to the fore in the process of merely preparing to do so. They are as revolutionary as one is used to from Einstein's brain children.
\end{abstract}

Keywords: - Going, back, Einstein, 1907, modern, Computer, Age

\section{(c) $\$(1)$}


(April 1 1 The first mechanical analog computer implementing special relativity and the "equivalence principle" based on it was invented by Einstein in his mind in 1907 [1], cf. [2]. It never got transformed into a hardware model. In the following we prepare the ground for this task. To our surprise, even in the preparation phase, EMAG (Einstein's Mental Analog Computer) produces several new predictions already which are amenable to empirical confirmation in continuation of Einstein's own list.

Einstein was thinking in pictures as is well known. To date, his pictorial imagination can be exactly reproduced in a computer environment. The equivalence principle between free fall on the one hand and a total lack of gravitation in outer space on the other led Einstein in 1907 to picturing in his mind a constantly accelerating long rocketship in outer space. He looked at an internal light ray being sent up from the bottom to the tip. And then he looked also at a light ray sent sideways across the middle of the rocketship, and the he imagined a light ray that hugs the bottom. After this latter achievement Einstein fell silent on the topic of gravitation for $3 \frac{1}{2}$ years. The unknown reason for the retreat is our topic in the following.

Einstein's mental image - his "happiest thought" as he always said - proved maximally fertile as one knows. As mentioned, he first imagined a light signal ascending vertically from the bottom up. It takes a fixed time to reach the tip since the distance is constant, the acceleration of the ship is constant, and $c$ is globally constant in special relativity which is assumed as the sole basis. During the light ray's travel time upwards, the whole ship including the tip has under the constant acceleration picked up a constant speed away from the point of origin of the light (namely, the point the bottom was at, at that very moment). The implied "Doppler effect" imposes a reduced frequency on the light sent up from the bottom when it arrives at the tip. This is the famous gravitational redshift that was discovered out of the blue sky by Einstein in 1907. The GPS confirms the slower ticking rate of clocks down on earth, compared to those in the satellites overhead, in our cars every day.

Einstein next considered also a horizontal light ray inside the vertically accelerating long rocketship. When sent sideways across the middle, it gets gently curved downwards. But when the light pulse is advancing along the horizontal bottom of the rocketship, with some glitter put in the air to make the light pulse visible towards above, then when looking down on it from the tip one necessarily finds it to be visibly slowed, as Einstein saw. A modern implementation consists in a "lightclock": a laser pulse going back and forth inside a glass tube with two mirrors at the ends and an appropriate energyrefreshing device on one side plus some glitter inside.

This proposal, made by Einstein in 1907 in somewhat less modern terms, is maximally revolutionary. It can be interpreted as the most demanding "spatiotemporal intelligence test." Even Einstein himself flunked his own test in part although everything he wrote was correct. Only the final conclusion to be drawn at the end, which is of special interest to us here, escaped his attention. And quantum mechanics which independently entails the same conclusion still lay two decades in the future.

What is this "final conclusion"? Whilst the light ray is horizontally progressing back and forth inside the light tube lying flat on the horizontal bottom of the constantly accelerating rocketship, the bottom itself is constantly falling back from the tip again even though keeping a constant distance as mentioned. Einstein correctly realized that the light pulse inside the glass tube when watched from the tip is bound to look slowed by the gravitational redshift factor. That is, by the very same factor by which every other clock downstairs is slowed. This finding looks like closing the case on the present mental experiment.

As it turns out, however, the case actually is far from being closed. For while the light pulse is advancing inside the horizontal light clock lying on the bottom, the bottom itself is continually falling back away from the tip at constant speed as we saw. What does this fact, re-encountered here, tell us? It means that the light pulse is propagating along a, with respect to the tip, constantly fallingback bottom as stated. That is, the locally horizontally moving light pulse actually proceeds in a slanted fashion relative to the tip! This twin implication to the gravitational redshift got overlooked. Einstein later confessed that he had "seared his mind" by thinking too hard and often about the spatiotemporal problem of the rocketship in his mental analog computer as one would say today. There were no computer simulations available at the time to check it all.

Even before such a rigorous simulation is going to be implemented, however, we have already accrued two results by now on sticking to Einstein's paradigm: First the mentioned slowdown of the light ray progressing across the bottom seen by Einstein. And second the just described slant. (Question: Is this slant pointing upwards or downwards? It does not matter but bets are welcome.) Both results - the familiar slowdown and the new slant - need to be taken together. The present task when seriously undertaken in one's mind almost overtaxes human imagination.

Everywhere locally, the light ray that is advancing horizontally across the bottom does so in a slanted fashion relative to the tip since the bottom is constantly falling away from the tip notwithstanding the constant distance kept, whilst the light pulse is progressing: Just as in the vertical case seen before jointly with Einstein. The new slant now directly explains why it is that the light ray looks slowed when watched from the tip as described by Einstein.

Note that if this new explanation of the "Einstein slowdown" is correct, the slowdown is no slowdown at all! For along the everywhere locally valid slanted motion relative to the tip, the speed of light $c$ is conserved. This finding jibes with the formal fact that the equivalence principle is exclusively based on special relativity with its by definition global $c$.

For some reason, the last-mentioned feature got overlooked, not only by Einstein himself in his own time but also by the scientific community for more than a century. Therefore, you as the reader should be pretty sure about whether you agree or not with what has been said above. For in case you agree, many things follow suit automatically because Einstein's brain children have a knack for entailing the impossible. 
The global $c$ just found retrieved downstairs is good for a revolution in physics if true. But it does not stand alone: Simultaneously, the path length covered by the advancing light pulse downstairs is increased relative to the tip by the gravitational redshift factor. This is because the present slant for once goes without the perspective-induced optical shortening that accompanies every other slanted transversal motion in nature. Note that special relativity "preserves lateral distances" in a receding frame. Therefore, the present slant is for once not accompanied by an optical shortening. Hence all distances downstairs and all objects downstairs are invisibly to the tip enlarged by the gravitational redshift factor. This new finding comes as a surprise. It means that Einstein's famous gravitational time dilation of 1907 has acquired a sibling: gravitational size dilation. The latter represents a new Einstein effect since the whole game is his invention.

Hence we are confronted here with another Einstein revolution: "All distances more downstairs in gravitation are increased in proportion to the relative clock slowdown." That is, all objects downstairs are invisibly to above enlarged by the gravitational redshift factor. This is a new fundamental result in physics, implicit in the equivalence principle and its generalizations.

The new-found result (global constancy of $c$ in the equivalence principle) extends to the Schwarzschild metric (where it was actually first vaguely spotted). If true, it enforces a "re-scaling" of general relativity as a whole. At the same time, it entails the existence of the long sought "holy grail of physics" (unification of quantum mechanics and general relativity). This new feature follows suit despite the fact that the full "global- $c$ transform of general relativity" remains to be written down.

Many things follow in the wake of the successfully restored global $c$ in the equivalence principle. For example, if the light from a black hole is infinitely redshifted as is indubitable, then the distance of the emitting "horizon" from the outside world is bound to be infinite, too, if $c$ is globally valid. Therefore, light can never reach the horizon of a black hole in finite outer-universe time. Hence there exists not a single "finished black hole" in the universe. And a fortiori, there exists no "interior solution" to the Schwarzschild metric and no "singularity" inside and no "Hawking radiation" at its boundary since there is no boundary.

A second surprising mega implication of "c-global retrieved" is that there exists "no Big Bang." For an expanding universe automatically involves superluminal recession speeds between sufficiently far apart elements. A third mega surprise is that there exist "no gravitational waves." A fourth is that micro black holes possess radically new properties besides staying unfinished forever, including the property of initial undetectability in the absence of Hawking radiation. It is for this reason that trying to produce micro black holes on earth (as CERN officially does for 11 years) is counter-advisable. All of this if taken together amounts to a revolution in physics being implicit in a crumb from Einstein's table: $c$ - $g$ lobal retrieved.

We are approaching the end. Is it scientifically legitimate to hark back so deep into the past of modern gravitation theory? Modern technology suggests it is high time to return to the "young David's" own workshop: to Einstein's painstakingly pictured extended long rocketship in outer space. For in our current computer age, the latter is readily amenable to simulation. From this insight it follows that the final truth is only a few simulation steps away. Hence if no mistake was made in the above attempt at better understanding Einstein's mental analog computer of 1907, an alternative title for the proposal made above could read "Einstein's IQ Test." The present authors share the reader's perplexity in the face of the attempted analogical thinking. The three of us first met in a 3semester course on the mentality of elephants in preparation for a film project. During our widely meandering discussions we at one point hit upon the above embarrassment of riches - or of nonsense, respectively - dependent on the reader's momentary mood. We hope that the present paper which if true radically changes the modern picture of the cosmos can be published as a precursor to a planned computer simulation.

\section{Acknowledgments}

OER thanks Wolfgang Müller-Schauenburg and Ali Sanayei for discussions. For J.O.R.

\section{References}

[1].A. Einstein, On the relativity principle and the conclusions drawn from it (in German). Jahrbuch der Radioaktivität 4, 411-462 (1907). English translation: http://www.pitt.edu/ jdnorton/teaching/GR\&Grav_2007/pdf/Einstein_1907.pdf

[2].O.E. Rossler, A footnote to Einstein 1907. European Scientific Journal 10 (33), 445-448 (2014). 